\title{
A roadmap for management of chronic thromboembolic pulmonary hypertension
}

\author{
To the Editor:
}

In a recent editorial published in the European Respiratory Journal, LANG and Matsubara [1] comment on the French experience of balloon pulmonary angioplasty (BPA) for inoperable chronic thromboembolic pulmonary hypertension (CTEPH) in the context of international BPA experience [2]. The groundbreaking development of BPA techniques (initially developed in the USA by FeINSTEIN et al. [3]) is widely recognised, and Matsubara and his Japanese colleagues should be congratulated for refining this treatment [4]. In the mid-2010s, BPA was implemented in high-volume expert centres in Europe, including France and Germany $[2,5]$. These large European centres host multidisciplinary teams with experienced surgeons for pulmonary endarterectomy, interventional radiologists/cardiologists for BPA, radiologists experienced in pulmonary vascular imaging and pulmonologists/cardiologists with expertise in pulmonary hypertension management [6]. As described in our recent article in the European Respiratory Journal [2], the French reference centre for pulmonary hypertension has generated strong data in a large cohort of inoperable CTEPH (or patients with persistent pulmonary hypertension after pulmonary endarterectomy) treated with BPA between 2014 and 2017. There is no doubt that BPA is an important treatment option for carefully selected CTEPH patients [1-6]. Some recommendations from LANG and Matsubara [1] are well taken and we agree that developing a BPA programme in an expert CTEPH centre is certainly a challenging but inspiring task and that our goals should be very ambitious. We wish, however, to provide some additional comments and ask to correct a number of erroneous statements in the editorial [1].

First, the statements on pulmonary endarterectomy should be further discussed. In France and in the UK, more than two endarterectomies are currently performed per million of the population in a single national referral centre in charge of treatment of the whole French and UK population, thanks to a structured network of commissioned centres [7, 8]. By contrast, in Japan, only 60 endarterectomies are performed annually in low-volume centres, with around 0.5 endarterectomies per million population (and not one per billion as originally stated in the editorial [1]). These differences should be elucidated. Among possible explanations, one could be lack of access to surgery or insufficient surgical expertise in some Japanese centres $[1,6]$. Moreover, it cannot be excluded that different CTEPH phenotypes may exist in Europe compared with Japan, with less proximal disease in Japan, as suggested by the editorialists [1,9]. Thus, indirect comparison between two different populations remains very hypothetical and subject to bias [1,9].

More concerning is table 1 in the editorial [1], which should be entirely revised due to multiple errors. We have tried to understand the numbers presented in the table and understand that there were some mistakes: 1) comparing lung injury per procedure in the Japanese study versus per patient in the French subjects undergoing several procedures $[1,2,4]$; 2) comparing long-term follow-up results in a subset of the Japanese multicentre registry versus results observed immediately post-BPA in all French patients undergoing BPA [1, 2, 4]; and 3) comparing incomplete haemodynamic data from both reports [1, 2, 4]. After correction of the above errors, we have produced a new table (table 1), describing the results in French and Japanese BPA programmes in terms of efficacy, safety and 30-day mortality.

There is no doubt that clinical outcomes of operable and inoperable CTEPH patients will improve markedly in the modern management era, and we are certainly on the way to near-normalisation of pulmonary haemodynamics at rest in most CTEPH patients treated in expert centres $[1,2,4,6]$. Multicentre consortia should now produce stronger data both from randomised controlled trials and multicentre registries, in order to implement CTEPH guidelines. It is clear that CTEPH management

@ERSpublications

Multicentre consortia should now produce stronger data both from randomised controlled trials and multicentre registries, in order to implement CTEPH guidelines http://bit.ly/2TWnaJ3

Cite this article as: Jaïs $\mathrm{X}$, Brenot $\mathrm{P}$, Montani $\mathrm{D}$, et al. A roadmap for management of chronic thromboembolic pulmonary hypertension. Eur Respir J 2019; 54: 1901295 [https://doi.org/10.1183/ 13993003.01295-2019]. 
TABLE 1 Results of balloon pulmonary angioplasty (BPA) in France and in Japan

French reference centre [2]

Japanese multicentre registry [4]

\begin{tabular}{|c|c|c|c|c|}
\hline Period & \multicolumn{2}{|c|}{ February 2014 to July 2017} & \multicolumn{2}{|c|}{ November 2004 to March 2013} \\
\hline Total procedures $\mathbf{n}$ & \multicolumn{2}{|c|}{1006} & \multicolumn{2}{|c|}{1408} \\
\hline Haemoptysis ( $\%$ of procedures) & \multicolumn{2}{|c|}{7.1} & \multicolumn{2}{|c|}{14} \\
\hline 30-day mortality $\%$ & \multicolumn{2}{|c|}{2.2} & \multicolumn{2}{|c|}{2.6} \\
\hline & Baseline & After BPA & Baseline & After BPA \\
\hline 6MWD m & $396 \pm 120$ & $441 \pm 104$ & Baseline values not & $401 \pm 105$ \\
\hline mPAP $\mathrm{mmHg}$ & $44 \pm 10$ & $32 \pm 9$ & presented in the & $24 \pm 6$ \\
\hline Cardiac index $L \cdot \min ^{-1} \cdot \mathrm{m}^{-2}$ & $2.7 \pm 0.6$ & $3.1 \pm 0.8$ & follow-up & $2.9 \pm 0.7$ \\
\hline PAWP $\mathrm{mmHg}$ & $10 \pm 3$ & $10 \pm 4$ & subpopulation & $7 \pm 3$ \\
\hline PVR dyn $\cdot s \cdot \mathrm{cm}^{-5}$ & $604 \pm 226$ & $329 \pm 177$ & & $360 \pm 223$ \\
\hline
\end{tabular}

Data are presented as mean $\pm \mathrm{SD}$, unless stated otherwise. PAH: pulmonary arterial hypertension; 6MWD: 6-min walk distance; mPAP: mean pulmonary artery pressure; PAWP: pulmonary artery wedge pressure; PVR: pulmonary vascular resistance.

requires high-volume centres and multidisciplinary approaches. In that context, BPA is a CTEPH treatment option among others [6]. Several studies are currently addressing key issues in this condition and their results are eagerly awaited. These include a better evaluation of the respective and potentially complementary roles of medical therapy and BPA in treatment-naïve patients with inoperable CTEPH (RACE study; NCT02634203). Moreover, an ongoing international BPA registry will provide long-term information on this procedure (NCT03245268). Lastly, it is very likely that medical, surgical and interventional approaches are not mutually exclusive and that CTEPH patients may benefit from multimodal "hybrid" treatment options.

Xavier Jaïs ${ }^{1,2,3}$, Philippe Brenot ${ }^{1,3,4}$, David Montani $\odot^{1,2,3}$, Elie Fadel ${ }^{1,3,5}$, Marc Humbert $\circledast^{1,2,3}$ and Gérald Simonneau ${ }^{1,2,3}$

${ }^{1}$ Univ. Paris-Sud, Faculté de Médecine, Université Paris-Saclay, Le Kremlin-Bicêtre, France. ${ }^{2}$ Inserm UMR_S 999, Hôpital Marie Lannelongue, Le Plessis Robinson, France. ${ }^{3}$ AP-HP, Service de Pneumologie, Centre de Référence de l'Hypertension Pulmonaire, Hôpital Bicêtre, Le Kremlin-Bicêtre, France. ${ }^{4}$ Service de Radiologie, Hôpital Marie Lannelongue, Le Plessis Robinson, France. ${ }^{5}$ Service de Chirurgie Thoracique, Hôpital Marie Lannelongue, Le Plessis Robinson, France.

Correspondence: Xavier Jaïs, Service de Pneumologie, Hôpital Bicêtre, 78 rue du Général Leclerc, 94275 Le KremlinBicêtre Cedex, France. E-mail: xavier.jais@gmail.com

Received: 30 June 2019 | Accepted: 17 Aug 2019

Conflict of interest: X. Jaïs reports grants and personal fees from Actelion, Bayer, GSK and MSD, outside the submitted work. P. Brenot has nothing to disclose. D. Montani reports grants and personal fees from Actelion, Bayer, GSK and MSD, outside the submitted work. E. Fadel has nothing to disclose. M. Humbert reports grants and personal fees from Actelion, Bayer, GSK and Merck, and personal fees from United Therapeutics, outside the submitted work. G. Simonneau reports grants, personal fees and non-financial support from Actelion, Bayer, GSK and Merck, outside the submitted work.

\section{References}

1 Lang IM, Matsubara H. Balloon pulmonary angioplasty for the treatment of chronic thromboembolic pulmonary hypertension: is Europe behind? Eur Respir J 2019; 53: 1900843.

2 Brenot P, Jaïs X, Taniguchi Y, et al. French experience of balloon pulmonary angioplasty for chronic thromboembolic pulmonary hypertension. Eur Respir J 2019; 53: 1802095.

3 Feinstein JA, Goldhaber SZ, Lock JE, et al. Balloon pulmonary angioplasty for treatment of chronic thromboembolic pulmonary hypertension. Circulation 2001; 103: 10-13.

4 Ogawa A, Satoh T, Fukuda T, et al. Balloon pulmonary angioplasty for chronic thromboembolic pulmonary hypertension: results of a multicenter registry. Circ Cardiovasc Qual Outcomes 2017; 10: e004029.

5 Olsson KM, Wiedenroth CB, Kamp JC, et al. Balloon pulmonary angioplasty for inoperable patients with chronic thromboembolic pulmonary hypertension: the initial German experience. Eur Respir J 2017; 49: 1602409.

6 Kim NH, Delcroix M, Jais X, et al. Chronic thromboembolic pulmonary hypertension. Eur Respir J 2019; 53: 1801915. 
7 Cannon JE, Su L, Kiely DG, et al. Dynamic risk stratification of patient long-term outcome after pulmonary endarterectomy: results from the United Kingdom National Cohort. Circulation 2016; 133: 1761-1771.

8 Amsallem M, Guihaire J, Arthur Ataam J, et al. Impact of the initiation of balloon pulmonary angioplasty program on referral of patients with chronic thromboembolic pulmonary hypertension to surgery. J Heart Lung Transplant 2018; 37: 1102-1110.

9 Chausheva S, Naito A, Ogawa A, et al. Chronic thromboembolic pulmonary hypertension in Austria and Japan. J Thorac Cardiovasc Surg 2019; 158: 604-614.e2.

\section{Balloon pulmonary angioplasty for the treatment of chronic thromboembolic pulmonary hypertension: is Europe behind?}

From the authors:

We are grateful to the editors to be able to correct an error in the table (the number for lung injury referred to \% of sessions) and one in the text ("billion" has been corrected to "million") of our editorial [1] accompanying the article by BRENOT et al. [2], published recently in the European Respiratory Journal. These errors have been corrected in the revised version of the table in the editorial; also see the author correction notice in this issue of the European Respiratory Journal.

We would also like to reply to the correspondence by X. Jaïs and co-workers, referring to our editorial, and further spotlight one of the most outstanding advances in pulmonary vascular treatments over the past decade.

We completely agree with $\mathrm{X}$. Jaïs and co-workers that balloon pulmonary angioplasty (BPA) is an important treatment option for carefully selected patients with chronic thromboembolic pulmonary hypertension (CTEPH), and that at present and in the future, medical, surgical and interventional approaches will be applied in combination to benefit patients and lead them to cure. What is important is that the aim of our editorial was to highlight the French data and comment on their relevance, while discussing important differences, particularly interventional and technical challenges, between European and Japanese patients.

Regarding "multiple errors" in the table, it must be said that BRENot et al. [2] state in their published manuscript that "All patients underwent a comprehensive clinical evaluation before the first BPA (baseline), before each BPA session and 3-6 months after the last BPA", and not "immediately post-BPA", as stated in the correspondence. Japanese follow-up data were collected after $425.5 \pm 280.9$ days [3] and those of the German series derive from after 24 weeks after the final BPA procedure [4].

Regarding incompleteness of haemodynamic data, we deliberately left out wedge pressure numbers as this opens another separate discussion on population-based differences between European and Japanese patients that we did not want to be the subject of our editorial.

Taken together, the French data showed a greater increase in cardiac output than the Japanese data. In European patients, BPA can decrease pulmonary vascular resistance (PVR) through a decrease of mean pulmonary artery pressure (mPAP), and a greater increase of cardiac output. In Japanese patients, BPA

@ERSpublications

Balloon pulmonary angioplasty is gaining ground in Europe

http://bit.ly/2m02zXF

Cite this article as: Lang IM, Matsubara H. Balloon pulmonary angioplasty for the treatment of chronic thromboembolic pulmonary hypertension: is Europe behind?. Eur Respir J 2019; 54: 1901639 [https://doi. org/10.1183/13993003.01639-2019]. 
can decrease PVR through a proportionally greater decrease of mPAP, with little increase of cardiac output.

Future research will be collaborative and shed light on unresolved issues of pulmonary vascular disease in CTEPH, for example the role of the bronchial circulation in CTEPH and the vascular biology of secondary pulmonary arteriopathy of CTEPH.

Irene M. Lang ${ }^{1}$ and Hiromi Matsubara ${ }^{2}$

${ }^{1}$ Dept of Internal Medicine II, Division of Cardiology, Vienna General Hospital, Medical University of Vienna, Vienna, Austria. ${ }^{2}$ National Hospital Organization, Okayama Medical Center, Okayama, Japan.

Correspondence: Irene M. Lang, Dept of Internal Medicine II, Division of Cardiology, Medical University of Vienna, Währinger Gürtel 18-20, 1090 Vienna, Austria. E-mail: irene.lang@meduniwien.ac.at

Received: 21 Aug 2019 | Accepted: 27 Aug 2019

Conflict of interest: I.M. Lang reports grants and personal fees from Actelion and AOP Orphan Pharmaceuticals AG, and personal fees from Ferrer, United Therapeutics, MSD and Medtronic, outside the submitted work. H. Matsubara reports personal fees from Actelion, AOP Orphan Pharmaceuticals AG, Bayer, GlaxoSmithKline, Pfizer Japan, Inc., United Therapeutics, Nippon Shinyaku, Co., Ltd and Kaneka Medix Corporation, outside the submitted work.

Support statement: This work was supported by the Austrian Science Foundation, grant F54 (to I.M. Lang). Funding information for this article has been deposited with the Crossref Funder Registry.

\section{References}

1 Lang IM, Matsubara H. Balloon pulmonary angioplasty for the treatment of chronic thromboembolic pulmonary hypertension: is Europe behind? Eur Respir J 2019; 53: 1900843.

2 Brenot $\mathrm{P}$, Jaïs $\mathrm{X}$, Taniguchi $\mathrm{Y}$, et al. French experience of balloon pulmonary angioplasty for chronic thromboembolic pulmonary hypertension. Eur Respir J 2019; 53: 1802095.

3 Ogawa A, Satoh T, Fukuda T, et al. Balloon pulmonary angioplasty for chronic thromboembolic pulmonary hypertension: results of a multicenter registry. Circ Cardiovasc Qual Outcomes 2017; 10: e004029.

4 Olsson KM, Wiedenroth CB, Kamp JC, et al. Balloon pulmonary angioplasty for inoperable patients with chronic thromboembolic pulmonary hypertension: the initial German experience. Eur Respir J 2017; 49: 1602409. 